\title{
One-step synthesis of Ag@PS nanospheres via flash nanoprecipitation
}

Xianxiang $\mathrm{Hu}{ }^{1,2}$ Yao Lu ${ }^{3}$, Rui Liu ${ }^{2 *}$, Lei Sun ${ }^{1}$, Ivan P. Parkin ${ }^{3}$, Xia Zhang ${ }^{1 *}$

${ }^{1}$ National \& Local Joint Engineering Research Center for Applied Technology of Hybrid Nanomaterials, Henan University, Kaifeng, 475004, china.

${ }^{2}$ Ministry of Education Key Laboratory of Advanced Civil Engineering Materials, School of Materials Science and Engineering, and Institute for Advanced Study, Tongji University, Shanghai, 201804, China.

${ }^{3}$ Nanoengineered Systems Laboratory, UCL Mechanical Engineering, University College London, London, WC1E 7JE, UK.

\section{Correspondence}

Xia Zhang, National \& Local Joint Engineering Research Center for Applied Technology of Hybrid Nanomaterials, Henan University, Kaifeng, 475004, china. E-mall:

xia.zhang@ucl.ac.uk.

Abstract: In this paper, we utilize a facile flash nanoprecipitation (FNP) process to generate Ag nanoparticles@nanosphere polymer composites. Ag nanoparticles can be loaded onto the polymeric polystyrene (PS) nanospheres surface by a simple and inexpensive method through in-situ growth; the amount of loaded Ag nanoparticles and the size of holes on polystyrene surface prepared by flash nanoprecipitation can be fine-tuned by changing the $\mathrm{pH}$ of the water cistern and the polystyrene nanoparticles should have a greater specific area. The one-step and successive preparation route for the as-synthesized Ag@PS composites nanoparticles show high catalytic ability in the reduction reaction of 4-nitrophenol. Importantly, the nanoparticles showed no loss catalytic performance over five cycles. 
Keywords: flash nanoprecipitation. Ag@polymeric polystyrene. nanoparticles. Catalytic

\section{1 | INTRODUCTION}

Metal nanoparticles with high surface-to-volume ratios have important applications in fields such as biomedical sciences, chemistry and engineering due to their unique properties. ${ }^{[1-3]}$ However, the function of these materials is often reduced due to instability, which leads to aggregation when the nanoparticles are in a bulk state. ${ }^{[4]}$ It is significant to develop a technique to prevent the aggregation of such metal nanoparticles. Polymers can be used as a stabilizer to support metal nanoparticles and also can increase the specific surface area. Liu groups have reported that gold or platinum nanoparticles can be uniformly coated on the block co-polymer surface of micelles formed from polystyrene-b-poly(4-vinylpyridine) (PS-b-PVP). ${ }^{[5-6]}$ Both Kim and Knag have shown that hydrophobic iron oxide or gold can be solubilized and conformably embedded in the hydrophobic polymer core of polystyrene-b-poly(acrylic acid) (PS-b-PAA). ${ }^{7-8}$

Self-assembly is an alternative method to incorporate inorganic materials onto a polymer

support matrix and is typically low-cost and environmentally benign. ${ }^{[7-8]}$ To obtain good hybrid inorganic/polymer nanocomposites, most of the self-assembly requires pre-synthesized nanoparticles with well-defined surfaces. Although some groups have reported "one-pot" methods to synthesize nanoparticles with the metal reduction and the formation of polymer colloids simultaneously, these methods are not readily scalable due to tedious reaction processes. ${ }^{[9]}$ Flash nanoprecipitation (FNP) is a useful self-assembly technique for producing uniform size nanoparticles. This is a process in which two high-speed linear jets of fluid (one containing the polymer solution and the other containing the non-solvent of the polymer) are mixed in a small chamber. Silver nanoparticles have attracted great interest in industry and academia because of their excellent catalysis, plasmonics, antimicrobial properties and sensing. ${ }^{[10-13]}$ In this work, we used a one-step flash nanoprecipitation method to fabricate Ag@PS microcomposite materials. Ag nanoparticles can be in-situ deposited onto the 
spherical polystyrene surface via electrostatic attractions. Interestingly, it was found that the polystyrene microsphere surface has many holes, which can increase the specific surface area. The obtained Ag nanoparticles decorated polystyrene microspheres exhibit excellent catalytic ability for the reduction of 4-nitrophenol via sodium borohydride reduction.

\section{2 | EXPERIMENTAL}

\section{1 | Materials}

Dodecanethiol, tetrabutylammonium bromide, acetone, caustic soda $(\mathrm{NaOH})$, carbon disulfide $\left(\mathrm{CS}_{2}\right)$, 2-bromopropionic acid, azobisisobutyronitrile (AIBN), styrene, methylbenzene, methylalcohol, sodium borohydride $\left(\mathrm{NaBH}_{4}\right)$, silver nitrate $\left(\mathrm{AgNO}_{3}\right)$ and sodium dodecyl sulfate (SDS) were purchased from Aladdin Chemistry Co. Ltd (Shanghai, China). All reagents were used as received without further purification and Milli-Q water with a resistivity of $18.2 \mathrm{M} \Omega$ was used in the experiments.

\section{2 | Synthesis of PS-COOH polymer}

Carboxyl functionalized polystyrene (PS-COOH) was obtained from surfactant-free emulsion polymerization (SFEP), in accordance with the referenced literature obtained polymers was used as the polymer support. ${ }^{16}$ In a typical synthesis, tetrabutylammonium bromide (1.93 g), $\mathrm{NaOH}(3.05 \mathrm{~g})$ and dodecanethiol $(18 \mathrm{~mL})$ were initially added to acetone $(240 \mathrm{~mL})$ and Milli-Q water $(30 \mathrm{~mL})$ in a three-neck flask under stirring for $30 \mathrm{~min}$ at room temperature, followed by cooled to $0{ }^{\circ} \mathrm{C}$ in an ice bath. Then $5.72 \mathrm{~g}$ of $\mathrm{CS}_{2}$ was dissolved in 2-bromopropionic acid $(6.75 \mathrm{~g})$ to form a mixture solution and was injected into the flask. The solution was heated to room temperature and stirred for $12 \mathrm{~h}$. Subsequently, the solution was bubbled with $\mathrm{N}_{2}$ for $30 \mathrm{~min}$ and then heated to $85{ }^{\circ} \mathrm{C}$, then AIBN $(0.125 \mathrm{~g})$ and styrene $(9 \mathrm{~g})$ were injected into the flask, the solution was then allowed to polymerize under reflux conditions and stirred for $24 \mathrm{~h}$. After the flask was put into an ice bath to cool down rapidly, the reaction was terminated. The mixture was dissolved in methylbenzene and methylalcohol was added to precipitate and purify the product. Finally, the sample was collected by vacuum filtration and dried at $50{ }^{\circ} \mathrm{C}$ under vacuum overnight. 


\section{3 | Synthesis of Ag@PS polymer colloids}

Fig. 1 shows the schematic process of preparation of Ag@PS polymer colloids. A syringe containing PS-COOH/THF solution $(1 \mathrm{~mL})$ at $3 \mathrm{mg} \cdot \mathrm{mL}^{-1}$ concentration was placed at the inlet of Stream 1, and another syringe containing $\mathrm{AgNO}_{3} / \mathrm{H}_{2} \mathrm{O}(1 \mathrm{~mL})$ at $0.5 \mathrm{mg} \cdot \mathrm{mL}^{-1}$ concentration was placed at the inlet of Stream 2. Then, the fluids from both syringes were expressed manually at the same rate $\left(\sim 1 \mathrm{~mL} \cdot \mathrm{s}^{-1}\right)$ to form a mixed solution. The mixed solution was diluted into a water cistern $(10 \mathrm{~mL})$ containing sodium dodecyl sulfate $(10 \mathrm{mg})$ and $\mathrm{NaBH}_{4}$ (0.1 mg, denoted as Sample 1, or $0.8 \mathrm{mg}$, denoted as Sample 2) under stirring for $1 \mathrm{~min}$ at room temperature. The product was collected by centrifugation and washed with Milli-Q water three times. Finally, the sample was dried at $50{ }^{\circ} \mathrm{C}$ under vacuum overnight.

\section{4 | Catalytic Study}

$2 \mathrm{mM}$ 4-nitrophenol $(2 \mathrm{~mL})$ was mixed with a freshly prepared aqueous solution of $\mathrm{NaBH}_{4}$ (4 mL, 0.2 M), After that, Ag@PS (1 mg) was added to the above solution. UV/Vis absorption spectra were recorded to monitor the change every minute, which take $50 \mu \mathrm{L}$ of the mixture diluted to $3 \mathrm{~mL}$ with Milli-Q water each time. After the reduction process was completed, the catalysts were centrifugally separated from the mixture and dried in a vacuum oven overnight for reuse in the next cycle-this process was repeated 5 times.

\section{5 | Characterizations}

Fourier Transform infrared spectroscopy (FT-IR) was carried out on a Magna-IRTM 550 spectrometer (Bruker Tensor 27, USA). Transmission electron microscopy (TEM) micrographs were collected using a JEOL JEM-2100F microscope equipped with a Gatan 894 Ultrascan 1k CCD camera (Japan) at a beam acceleration of $200 \mathrm{kV}$. Dynamic light scattering (DLS) were carried out on a nanoparticle analyzer SZ-100 (HORIBA Scientific, Japan). Zeta potentials of nanoparticle samples were obtained using the same instrument (HORIBA Scientific, Japan). X-ray diffractometer (XRD) was characterized by a Bruker D8 Advance $\mathrm{X}$-ray powder diffractometer (Germany) using $\mathrm{Cu} \mathrm{K \alpha}$ radiation $(\lambda=0.15418 \mathrm{~nm})$. UV-Vis spectra were recorded using an Evolution 220 recording spectrophotometer (Thermo fisher, America). 


\section{3 | RESULTS AND DISCUSSION}

The basic concept of the proposed process for constrained-volume synthesis of Ag@PS nanocomposite polymer colloids is shown in Fig. 1. It is composed of three assembly units including polymer, solvent, and a non-solvent solution. The interior structure is a T-shape mixer, which is employed to cause the two streams to merge, the details of design and fabrication are described by Zhang and Han. ${ }^{[14-15]}$ The streams are then diluted into a water reservoir that included the reducing agent $\left(\mathrm{NaBH}_{4}\right)$, stabilizer, and sodium dodecyl sulfate (SDS). During this flash nano-precipitation process, the PS-COOH polymer solution was mixed with the non-solvent solution (water) within a few milliseconds. Then the rapid mixing induced self-assembly of the polymer and $\mathrm{Ag}^{+}$combined with polystyrene nanoparticles through electrostatic interaction. Ag@PS nanoparticles are formed through an in-situ method, which facilitates the reduction reaction. Meanwhile, the remaining carboxyl group on the $\mathrm{Ag} @ \mathrm{PS}$ nanoparticles are neutralized to carboxylate by the $\mathrm{BH}_{4}{ }^{-}$and hydrolyzed by the water reservoir, because the carboxylate is strongly hydrophilic; this results in the microparticles swelling and leaving the holes. The water reservoir kinetically freezes the nanoparticles. ${ }^{[16-17]}$ When the $\mathrm{NaBH}_{4}$ content increased from 0.1 to $0.8 \mathrm{mg}$ in the water cistern, the $\mathrm{pH}$ increases from 8.34 to 9.14 , which makes more $-\mathrm{COO}^{-}$groups and leaves more deep holes. This gives more locations for $\mathrm{Ag}^{+}$to land on the polystyrene microparticles. The Zeta-potential of sample 1 and sample 2 are $-29.7 \mathrm{mV}$ and $-123.1 \mathrm{mV}$ in water $(\mathrm{pH}=7)$, respectively.

Fig. 2 shows the FT-IR spectrum of the PS-COOH polymer. The band at $3026 \mathrm{~cm}^{-1}$ was attributed to the aromatic C-H stretching vibrations, and the band at 754 and $696 \mathrm{~cm}^{-1}$ was attributed to the aromatic $\mathrm{C}-\mathrm{H}$ bending vibrations, whereas aromatic $\mathrm{C}=\mathrm{C}$ stretching vibrations appeared at 1601, 1493 and $1452 \mathrm{~cm}^{-1} .^{[18]}$ The distinct absorption peaks at $1735 \mathrm{~cm}^{-1}$ and 1290 $\mathrm{cm}^{-1}$ can be attributed to the $\mathrm{C}=\mathrm{O}$ stretching and the $\mathrm{C}-\mathrm{O}$ stretching of the carboxyl group, respectively. In addition, a broadening of the band due to $\mathrm{O}-\mathrm{H}$ stretching vibrations of the carboxyl group at $3422 \mathrm{~cm}^{-1}$ was observed, ${ }^{[19-20]}$ suggesting that the carboxylic groups were successfully modified on the polymer, which is useful for the deposition of Ag nanoparticles in the next step. Fig. 3 demonstrates the X-ray diffraction (XRD) pattern of the obtained polystyrene microparticles and Ag@PS microparticles. The XRD pattern of non-crystallized 
polystyrene microspheres was characterized by a broad peak at a $2 \theta$ value of $20^{\circ}$. And the broad peak comes from the polystyrene microparticles. From the XRD pattern of Ag@PS microparticles, it can be observed that all the diffraction peaks can be indexed to the face-centred cubic (fcc) structure of Ag, which matches well with the reported data (the Standards International Center for diffraction data JCPDS card no. 04-0783). The results exhibit that there are four obvious peaks at $38^{\circ}, 44^{\circ}, 64^{\circ}, 77^{\circ}$, and $81^{\circ}$, which matched well up to the (111), (200), (220), (311), and (222) planes of the silver metal. Note that the broad peak at about $20^{\circ}$ comes from non-crystallized polystyrene microspheres. No peaks from other phases were detected, indicating that the products contained no other detectable crystalline impurities.

Fig. 4(A) and Fig. 4(B) shows TEM images of the Ag@PS microparticles of Sample 1 and Sample 2. It could be seen that the microparticle surface decorated with many nanoparticles which size is about $8-15 \mathrm{~nm}$. Note that there are many holes on the microparticle surface, which can increase the specific surface area. From Fig. 4(B), it can be seen that the size of the microparticles is about $210 \mathrm{~nm}$ and there are also many holes on the surface. The density of Ag nanoparticles decorated on Sample 2 surface is large than that on Sample 1 surface because of the different $\mathrm{NaBH}_{4}$ concentration, which indicates tunable catalytic performance. SEM image (Fig.4C) of the microparticles from Sample 2 demonstrates that there are a lot of holes on the surfaces and the size of the microparticles are about $210 \mathrm{~nm}$, which is consistent with the TEM observation. Fig. 4(D) shows the HRTEM image of Ag nanoparticles decorated on the surface; the Ag nanoparticle is crystalline and the $2.32 \AA$ lattice spacing corresponds to the Ag (111) crystal plane of face-centered cubic (fcc) structure.

Dynamic light scattering (DLS) data (Fig. 5A) shows that the microparticles were well dispersed in water and have a narrow size distribution. The hydrodynamic diameter of Sample 1 and Sample 2 is about $200 \mathrm{~nm}$ and $210 \mathrm{~nm}$, which correspond with the TEM analysis. The UV-Vis absorbance of the colloids was measured and investigated for the surface plasmon resonance (SPR) peak associated with the presence of Ag nanoparticles. Photographs of colloidal solutions (inset in Fig. 5B) show that the obtained samples were well dispersed in water as Sample 1 was coloured yellow while Sample 2 was coloured dark green. The 
spectrum of the colloids at $0.5 \mathrm{mg} \cdot \mathrm{mL}^{-1}$ feed concentration in Fig. 5(B) shows an obvious plasmon band, indicating a continuous distribution of $\mathrm{Ag}$ nanoparticles. At a $\mathrm{AgNO}_{3}$ feed concentration of $0.5 \mathrm{mg} \cdot \mathrm{mL}^{-1}$, an intense plasmon absorption band with the maximum absorption peak of around $390 \mathrm{~nm}$ is observed. An increase in the concentration of $\mathrm{NaBH}_{4}$ products, a more intense plasma absorption band, and this results from more Ag nanoparticles being loaded.

Metal@polymer nanocomposites have been exploited for a number of advanced functional applications, such as electronic devices, sensors, catalysts and drug carriers. ${ }^{[21]}$ Catalytic reduction of 4-nitrophenol has been investigated by different groups, and now this reaction has become one of the model reactions for evaluating the catalytic activity of noble metal nanoparticles. Herein, we also study the silver-catalyzed reduction of 4-nitrophenol by $\mathrm{NaBH}_{4}$ to 4-aminophenol. As shown in Fig. 6(A), after catalyzed reduction, the colour of the solution turned from yellow to colourless. Fig. 6(B) and Fig. 6(C) show the comparison between Sample 1 and Sample 2 into the 4-nitrophenol solution. The intensity of the absorption peak at $400 \mathrm{~nm}$ decreased, together with the appearance of a new peak at $295 \mathrm{~nm}$, which was due to the gradual conversion of 4-nitrophenol to 4-aminophenol in the presence of $\mathrm{Ag} @ \mathrm{PS}$ nanoparticles. The degradation reactions were complete in $\sim 8 \mathrm{~min}$ and $\sim 4 \mathrm{~min}$, respectively. The reaction rate is faster than that of the reported silver nanoparticles on cellulose nanocrystals and Ag nanoparticles immobilized on spherical polyelectrolyte brushes. ${ }^{[22-23]}$ The nanocatalysts also exhibited good reusability for 4-nitrophenol reduction without any detectible loss in activity after 5 times re-use and retained the conversion close to $100 \%$ within $4 \mathrm{~min}$ of reaction time (Fig.6C, inset). Clearly, the composites with $\mathrm{Ag}$ nanoparticles embedded on the polymer particles surface are effective at preventing the aggregation of catalyst nanoparticles, making the catalyst reusable after multiple cycles of reactions. The kinetic data can be fitted to a Langmuir - Hinshelwood apparent first-order plot. ${ }^{[2]}$ The rate constant $\mathrm{k}$ was determined from the linear plot of $\ln \left(\mathrm{C}_{\mathrm{t}} / \mathrm{C}_{0}\right)$ versus time $\mathrm{t}$ (minutes), its value was determined to be $0.27 \mathrm{~min}^{-1}$ for Sample 1 and $0.54 \mathrm{~min}^{-1}$ for Sample 2 (see Fig. 6D). Because of the advantages of the facile synthesis, flash nanoprecipitation is an attractive platform for the production of stable and recyclable nanocatalysts. 


\section{4 | CONCLUSIONS}

In conclusion, we demonstrate a one-step and continuous method to synthesize Ag@PS composites via flash nanoprecipitation. The hybrid nanoparticles Ag@PS exhibited high catalytic performance to p-nitrophenol. This simple and versatile synthetic approach could be applied for a large-scale production of various nanocomposites by the continuous and low-energy FNP. This strategy could also be used in the fields of medication, energy and environmental.

\section{REFERENCES}

[1] D. Astruc, F. Lu and J. R. Aranzaes, Angew. Chem. Int. Ed. 2005, 44, 7852.

[2] H. B. Na, I. C. Song and T. Hyeon, Adv. Mater. 2009, 21, 2133.

[3] Y. Xu, L. Chen, X. Wang, W. Yao, Q. Zhang, Nanoscale. 2015, 7, 10559.

[4] C. Burda, X. Chen, R. Narayanan and M. A. El-Sayed, Chem. Rev. 2005, 105, 1025.

[5] R. Liu, C. Sosa, Y. W. Yeh, F. Qu, N. Yao, R. K. Prud'homme, R. D. Priestley, J. Mater. Chem. A 2014, 2, 17286.

[6] Y. Z. He, B. J. Wang, X. X. Hu, X. Zhang, L. Sun, R. D. Priestley, R. Liu, Colloid Polym. Sci. 2017, 295, 521.

[7] B. S. Kim, J. M. Qiu, J. P. Wang and T. A. Taton, Nano Lett. 2005, 5, 1987.

[8] Y. Kang and T. A. Taton, Angew. Chem. Int. Ed. 2005, 117, 413.

[9] R. Shenhar, T. B. Norsten and V. M. Rotello, Adv. Mater. 2005, 17, 657.

[10] M. Grzelczak, J. Vermants, E. M. Furst and L. M. Liz-Marzan, ACS Nano. 2010, 4, 3591.

[11] H. Tanaka, S. Koizumi, T. Hashimoto, H. Itoh, M. Satoh, K. Naka and Y. Chujo, Macromolecules 2007, 40, 4327.

[12] S. Prabhu, K. P. Eldho, Effects. Int. Nano Lett. 2012, 2, 32. 
[13] J. Tian, R. Liu, G. Wang, Y. Xu, X. Wang and H. Yu, Appl. Surf. Sci. 2014, 319, 324.

[14] Q. Zhang, J. Ge, T. Pham, J. Goebl, Y. Hu, Z. Lu, Y. Yin, Angew. Chem. Int. Ed. 2009, 48,3516 .

[15] C. Gao, Z. Lu, Y. Liu, Q. Zhang, M. Chi, Q. Cheng, Y. Yin, Angew. Chem. Int.Ed. 2012, 51, 5629 .

[16] C. Zhang, V. J. Pansare, R. K. Prud'homme, R. D. Priestley, Soft Matter. 2012, 8, 86.

[17] J. Han, Z. Zhu, H. Qian, A. R. Wohl, C. J. Beaman, T. R. Hoye, C. W. Macosko, J. Pharm. Sci. 2012, 101, 4018.

[18] M. Okubo, A. Ito, T. Kanenobu, Colloid Polym. Sci. 1996, 274, 801.

[19] M. Okubo, A. Ito, A. Hashiba, Colloid Polym. Sci. 1996, 274, 428.

[20] A. Kausar, S. Zulfiqar, M. I. Sarwar, J. Appl. Polym. Sci. 2014, 131, 39954.

[21] N. Y. Wang, Y. Liu, Z. W. Qiao, L. Diestel, J. Zhou, A. S. Huang and J. Caro, J. Mater. Chem. A 2015, 3, 4722.

[22] Z. N. Li, C. J. Wu, K. Zhao, B. Peng and Z. W. Deng, Colloids Surf. A 2015, 470, 80.

[23] B. Q. Mao, Q. D. An, B. Zhai, Z. Y. Xiao and S. R. Zhai, RSC Adv. 2016, 6, 47761.

[24] J. T. Tang, Z. Q. Shi, R. M. Berry, and K. C. Tam, Ind. Eng. Chem. Res. 2015, 54, 3299.

[25] J. J. Liu, J. Wang, Z. M. Zhu, L. Li, and X. H. Guo, AIChE journal. 2014, 60, 1977.

[26] Q. Geng, J. Du, RSC Adv. 2014, 4, 16425. 


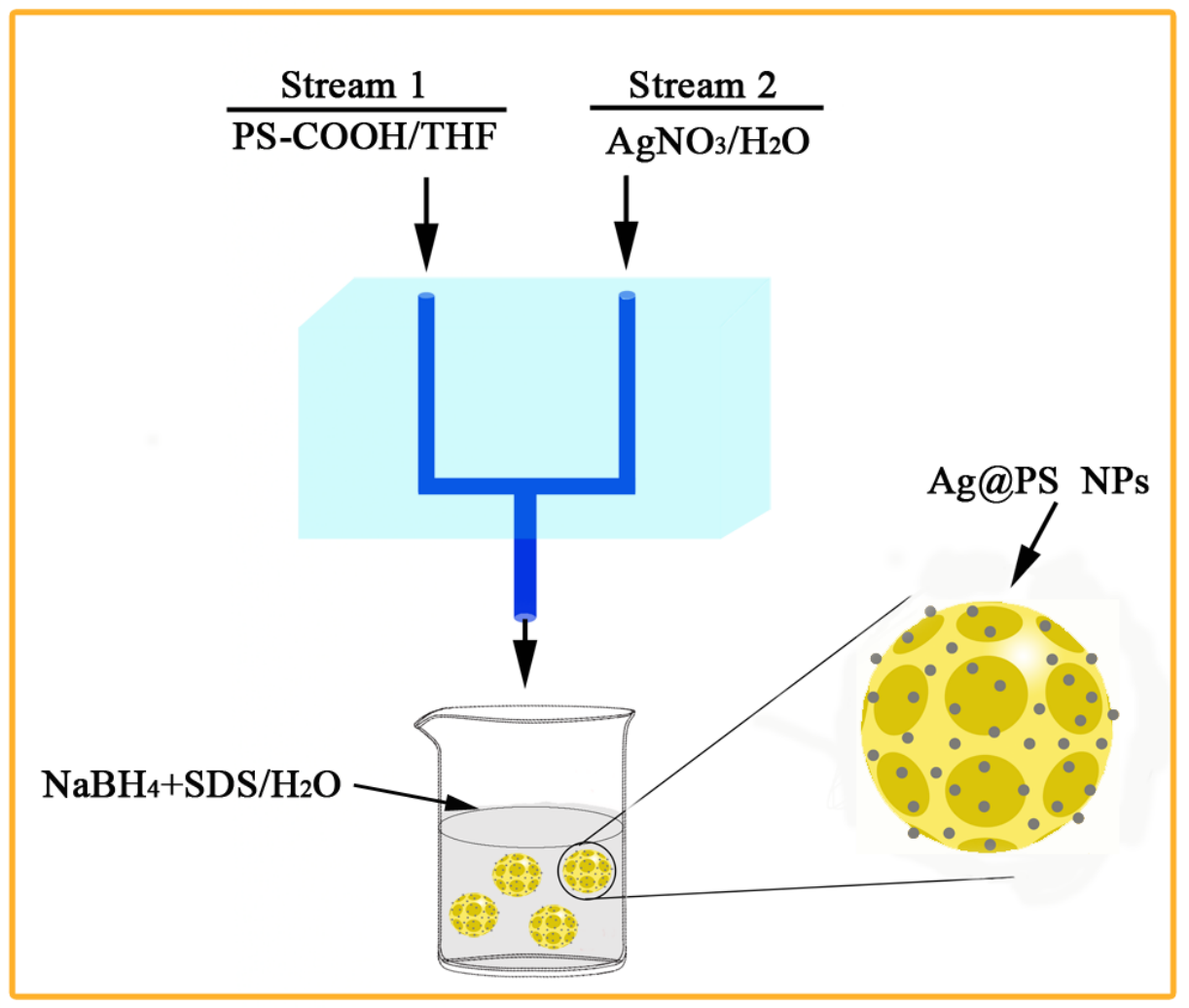

Fig. 1

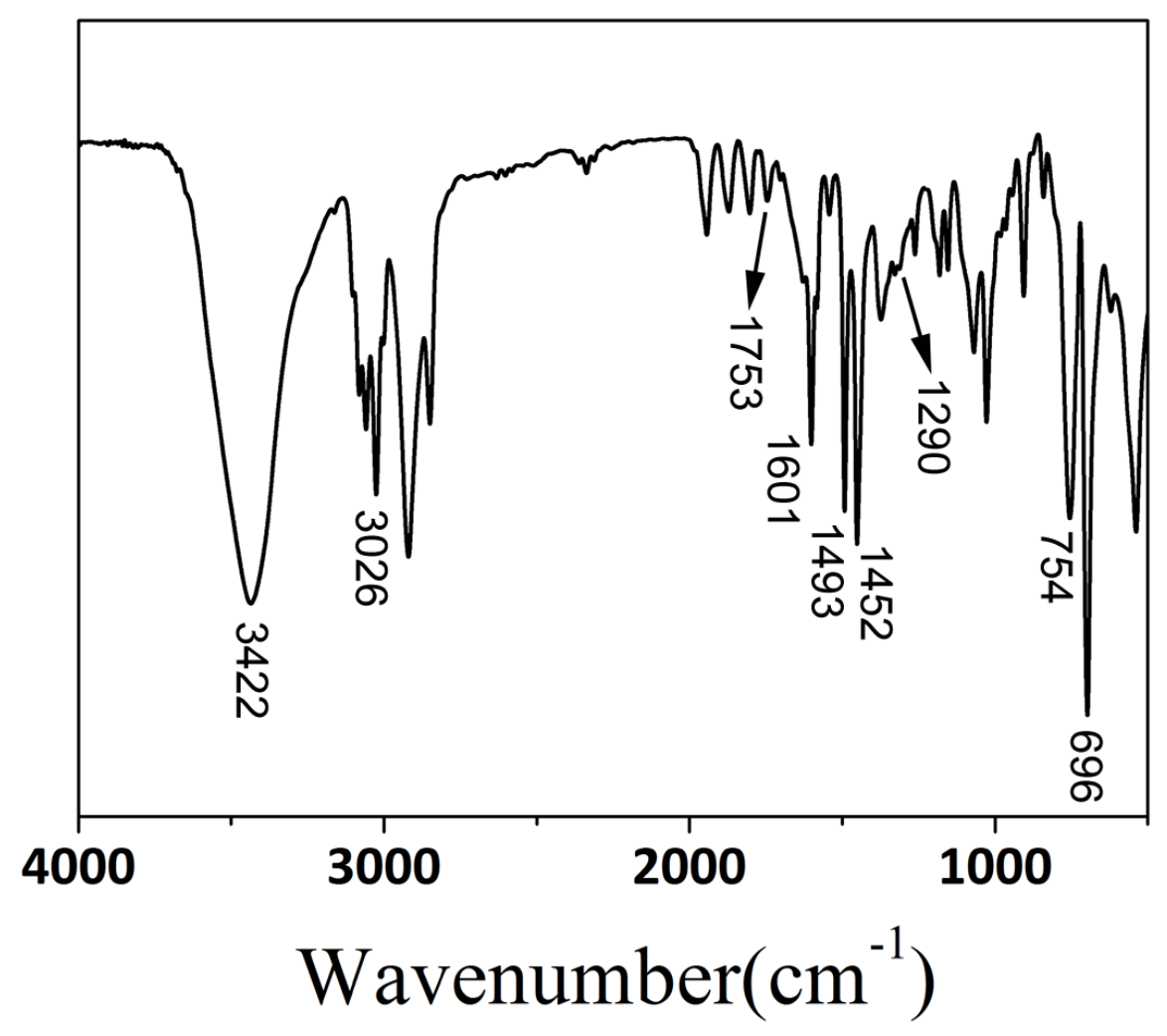

Fig. 2 


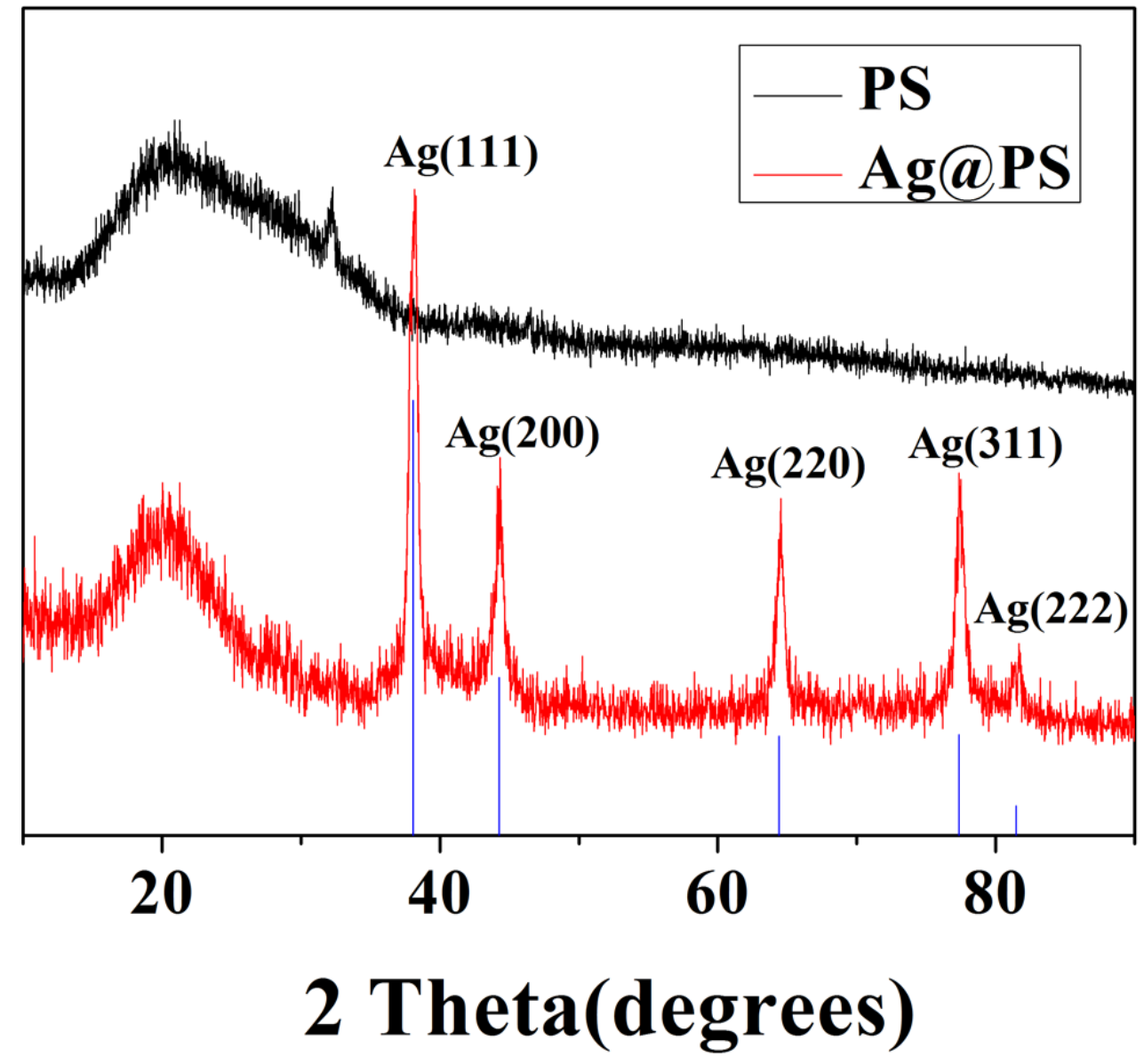

Fig. 3 


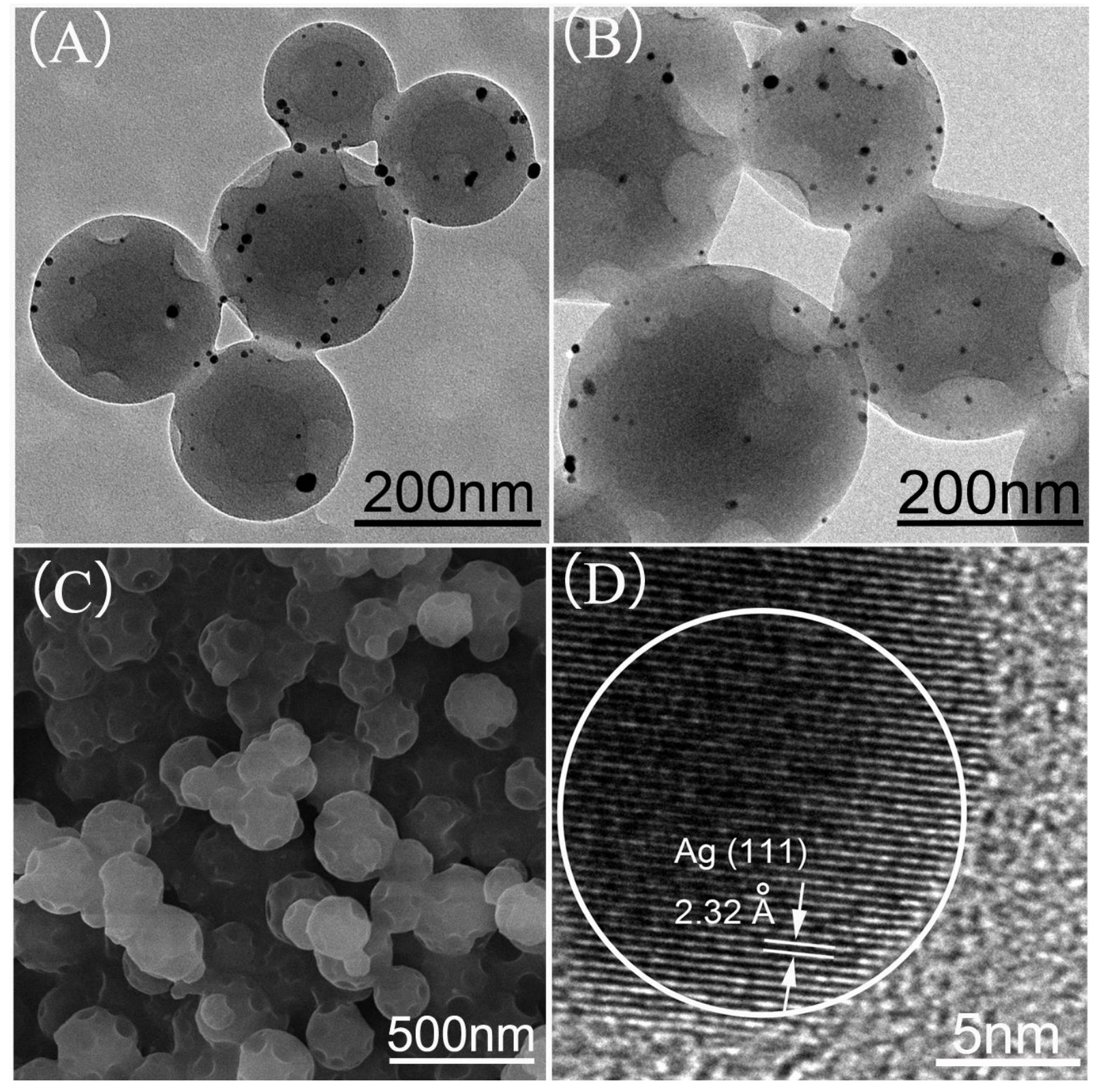

Fig. 4 

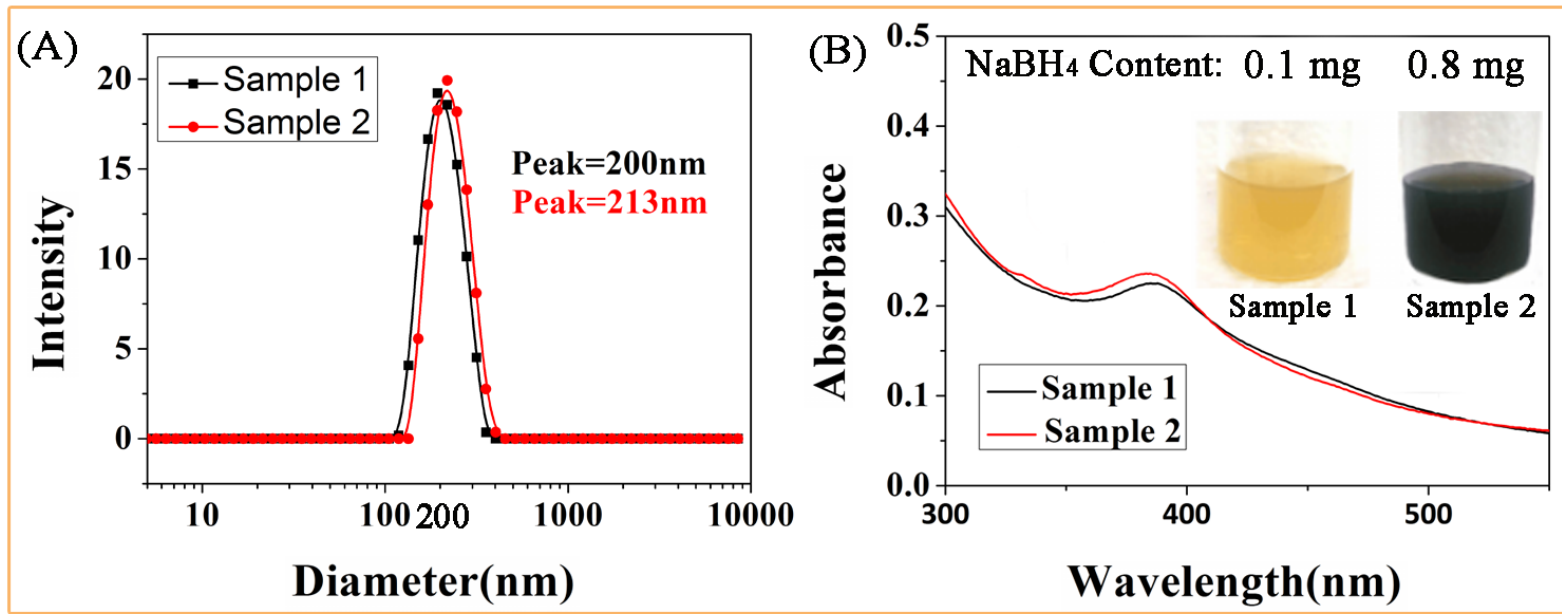

Fig. 5

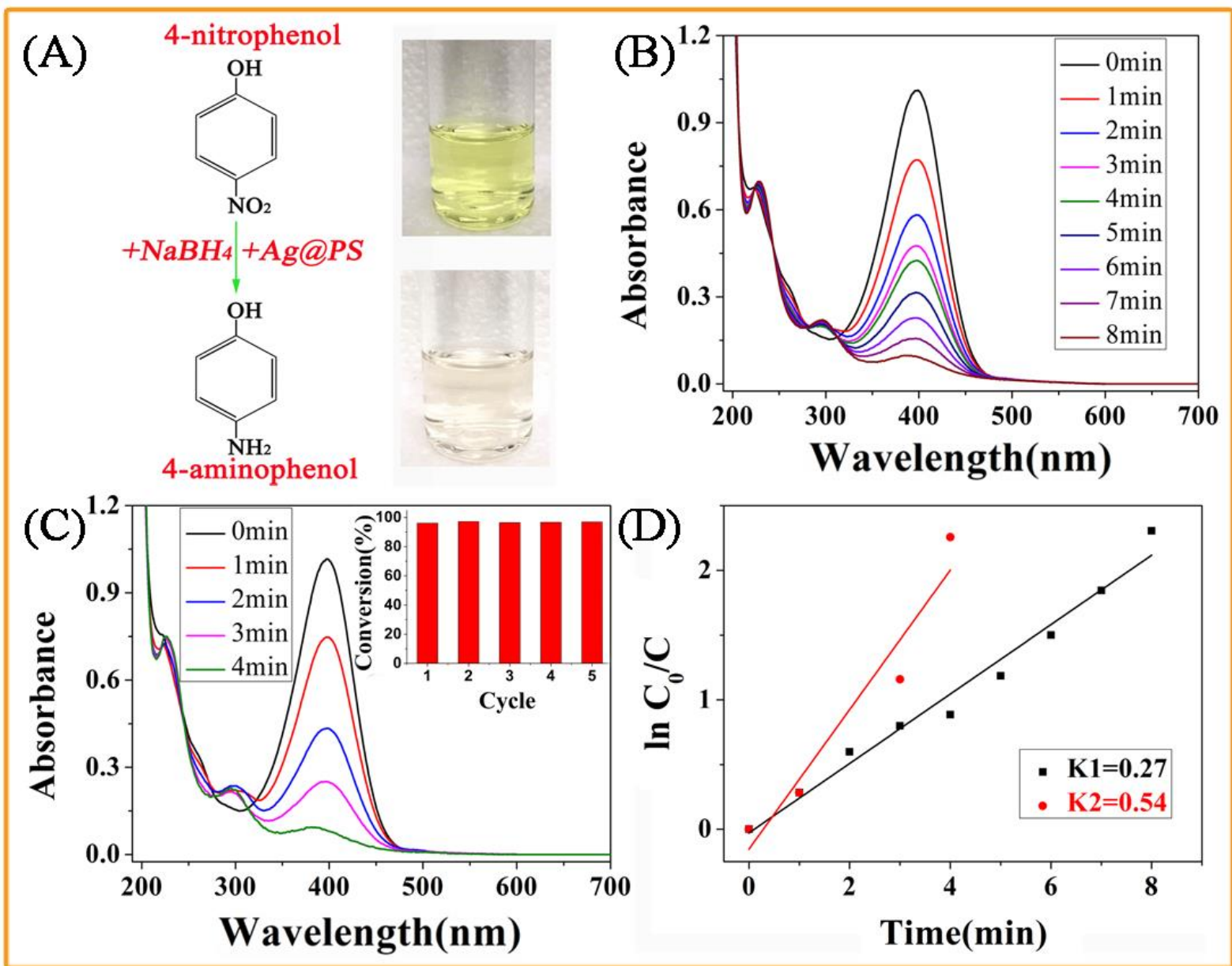

Fig. 6 